\title{
Perioperative booklet for orthognathic patients: A randomized controlled clinical trial
}

\author{
Cristina Silva Sousa*, Vanessa de Brito Poveda, Ruth Natalia Teresa Turrini \\ School of Nursing, Department of Medical-Surgical Nursing, University of São Paulo, São Paulo, Brazil
}

Received: May 22, 2018

DOI: $10.5430 /$ jnep.v8n12p90

\author{
Accepted: August 7, 2018 \\ Online Published: August 20, 2018
}

\begin{abstract}
Objective: This study compared the effectiveness of an informational booklet on postoperative self-care, knowledge, anxiety and symptoms related to orthognathic surgery.

Methods: This study is a randomized, single-blind, controlled clinical trial. This study was carried out from August 2013 to August 2015 and included 40 participants (20/group). The intervention group had routine postoperative surgeon guidance, and received a self-care booklet. Booklet information was explained by a nurse. The control group received only routine surgeon guidance. Knowledge and anxiety were compared pre- and postoperatively. Postoperative outcomes included self-care (oral hygiene, mobility and sensitivity, hydration of the lips, edema, and sleeping and breathing conditions), nutrition (eating ability and change in bodyweight), and pain (treatment required and a visual analog scale). Data were analyzed by general linear mixed models and mixed-effects models.

Results: Knowledge was higher in intervention group compared to control group $(p<.001)$. Anxiety assessment displayed no difference between groups. The intervention group had more halitosis $(p=.003)$ and greater bodyweight loss $(p=.002)$.

Conclusions: The booklet increased knowledge of postoperative self-care, but did not lower anxiety. Halitosis and weight loss were higher in the intervention group, however, most outcomes were similar between groups.
\end{abstract}

Key Words: Perioperative nursing, Clinical trial, Orthognathic surgery, Booklet

\section{INTRODUCTION}

Orthognathic surgery is performed in order to correct malocclusion and avoid bad effects of mastication, joint function, airway permeability and facial aesthetics. ${ }^{[1]}$

There is a lack of studies about the effectiveness of educational strategies for patients undergoing orthognathic surgery. Nurses routinely communicate and interact with patients for information and guidance of their individual needs, and they may be asked to implement current interventions to enhance patients' postoperative understanding of self-care expectations. $^{[2]}$

In systematic review on pre-operative orthopaedic patient education, authors discussed 11 studies that involved educational interventions among 1,044 participants. Interventions were based on an analysis of booklet or a booklet in combination with other teaching strategies. ${ }^{[3]}$ The most common outcomes related to pain, knowledge, anxiety, exercises and length of hospital stay. Based on the findings of this study, preoperative education seems to have some impact on patients' anxiety and knowledge levels. However, there is a necessity of more research, for suggestions about clinical practice to be made. ${ }^{[3]}$

Systematic review defines preoperative education as any educational intervention delivered before surgery that aims to

*Correspondence: Cristina Silva Sousa; Email: crissousa@usp.br; Address: Enéas de Carvalho Aguiar, São Paulo, SP, Brazil. 
improve patients' knowledge, health behaviors, and health outcomes. ${ }^{[4]}$ Preoperative education has been associated with decreased pre- and post-operative anxiety and pain, better coping, shorter hospital length of stay, lower readmissions, and lower costs. ${ }^{[5,6]}$

In orthognathic surgery, the postoperative period is the longest stage of the perioperative period. Preparation for experiencing this stage starts before surgery, when patient begins orthodontic treatment in preparation for the surgery ( 8 to 12 months prior). Patients need to understand postoperative self-care. Greater knowledge about the surgical procedure and postoperative care will impact positively self-care. Our aim was compare the effectiveness of an informational booklet on postoperative self-care, knowledge, anxiety and symptoms related to orthognathic surgery.

\section{METHODS AND MATERIAL}

\subsection{Study design}

This study was a randomized, single-blinded, controlled clinical trial carried out from August 2013 to August 2015. The intervention group received a booklet provided by a nurse and routine oral care information from the surgeon. Control group participants received only the routine oral care information from the surgeon.

\subsection{Setting and sample}

The setting was an Outpatient Clinic of Oral and Maxillofacial Surgery in Sao Paulo, Brazil. Forty-nine potential participants were assessed. Eight participants discontinued treatment, and one participant chose not to participate in the study. A total of 40 participants were randomly allocated into groups. Randomization was performed with a randomization block. Each block contained 4 random treatment assignments. After study subjects were allocated, there was not lost follow up through the 45-day postoperative period (see Figure 1).

Sample size was calculated on number of orthognathic surgery performed by the surgical team in one outpatient clinic. The power of the test, calculated upon completion of data collection, was $99.9 \%$.

Participants were included if they were scheduled to undergo orthognathic surgery (surgical techniques: bilateral sagittal split osteotomy of the mandibular ramus; intraoral vertical ramus osteotomy; Le Fort I osteotomy, and combined osteotomy, with or without mentoplasty or maxillary disjunction). Participants undergoing additional surgeries after orthognathic surgery and those with cleft lip and palate were excluded because these patients require other postoperative care.

Published by Sciedu Press

\subsection{Ethical consideration}

This study was approved by the Research Ethics Committee by University of Sao Paulo, Brazil, under opinion No. 193,454/13. Participants provided written informed consent prior to enrollment. The clinical trial was registered at the National Institutes of Health (NIH) - ClinicalTrials.gov NCT01803204.

\subsection{Randomization and blinding}

Participants were assigned to different blocks. In randomization block, each block contains a predetermined number of participations and the order of treatments is randomly exchanged within a block. Each block contained four participants' random treatment assignments. The random exchange of the segments ensured balance in the number of individuals allocated to each arm of the study. ${ }^{[7]}$

Two researchers were involved in conducting the study. The first researcher was responsible for participant enrollment, obtaining informed consent, and providing the intervention. The second researcher, who was blinded to group assignment, was responsible for data collection of outcomes and for examining participants during postoperative follow-up.

\subsection{Intervention}

The intervention consisted of a booklet and an oral guidance by a nurse. Booklet "Orthognathic Surgery for Patients" included information about orthognathic surgery, preoperative care, hospital routine until discharge, possible surgical complications, postoperative care and frequently asked questions (FAQ). ${ }^{[8]}$ Oral guidance consisted of advising the participant about oral hygiene (use of brush with soft bristles or child size, importance of performing brushing with a brush, not just mouthwash with antiseptic), type and consistency of diet (liquid diet, pasty, soft foods, and normal food according to the postoperative day), facial exercises (smile, moving air in the cheeks, passing the tongue on the lips), edema control (use of ice pack, keeping head elevated longer), avoiding sun exposure, lip care (use of lip hydration, maintaining oral hydration), dressing, and postoperative rest (using two pillows on the bed for head elevation, and holding the head a little more elevated throughout the day in order to better breathing).

The booklet was written by the perioperative nurse researcher. Booklet content was based on findings from groups of patients in postoperative period, searches of patients' comments in virtual environments (blogs), and oral surgeon guidance, nurse specialists, speech therapists, and nutritionists. ${ }^{[8]}$ Booklet was validated by a multidisciplinary team of 10 professionals (4 maxillofacial surgeons, 2 nurses, 2 nutritionists and 2 speech therapists) using the Delphi technique ${ }^{[9]}$ for 
content validity, assessment, language, illustrations, layout, validated by 20 patients undergoing orthognathic surgery. motivation, and culture. In the third round, the Delphi measurement was 0.972 and the interclass correlation coefficient was $0.601(p<.001) .{ }^{[9]}$ Subsequently, booklet content was

Participants assessed content relevance related to the content itself, content organization, writing, and appearance. Content validity coefficient (CVC) was $0.90 .^{[8]}$

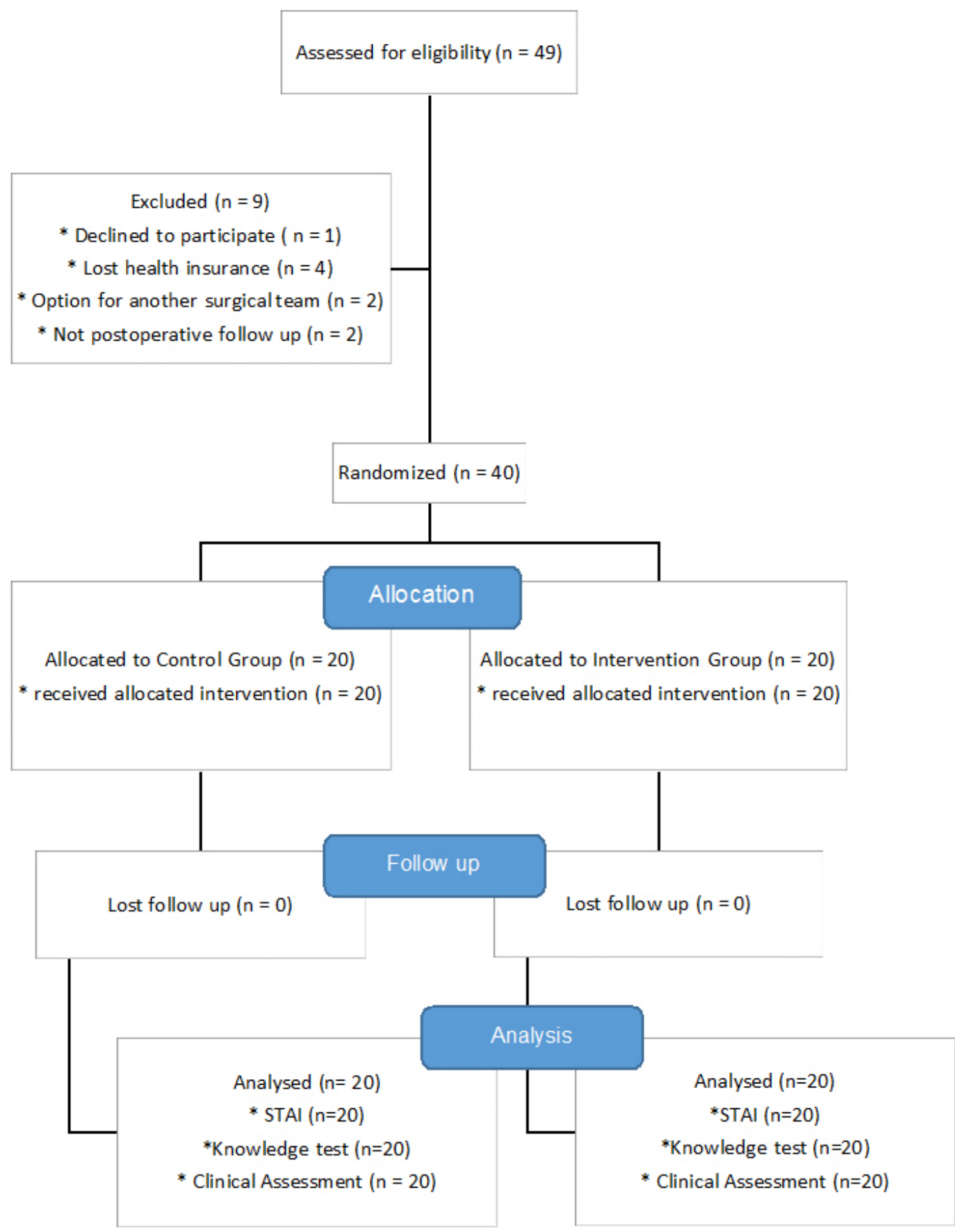

Figure 1. Flow diagram

The intervention and control groups had access to the information provided from surgeons during the preoperative consultation. Information provided by surgeons, included guidance about surgery, fasting, preoperative examinations, postoperative edema, presence of paresthesia, limited mouth opening, possible hematoma, changes in facial aesthetics, and information about the postoperative period (oral hygiene, limited opening of the oral cavity, careful manipulation of 
orthodontic elastics, and control of postoperative pain). At the end of the study, the booklet was offered to the control group.

\subsection{Outcomes and measurement}

An investigator-developed demographic assessment included six items (i.e. gender, age, bodyweight, height, marital status, scholarly level), and three variables (i.e. obesity, diabetes, and smoking). Additionally, participants responded to questions about motivation for surgery (four options: functional improvement, aesthetic improvement, medical indication, own initiative), and expectations for surgery (four options: social/affective, physical/aesthetic, professional, and personal satisfaction).

Anxiety levels were measured using the 20-item self-report State-Trait Anxiety Inventory (STAI). This instrument assesses two anxiety dimensions, transitional or state anxiety trait and anxiety that reflects a relatively stable condition of a patient's anxiety. Items from this scale were rated on a 4-point Likert scale, with greater scores indicating more trait anxiety. ${ }^{[10]}$

Knowledge level about the surgery and postoperative care involved completion of 10 questions with four multiple choice response options. A correct answer to a question added 1 point to the score; incorrect answers and "don't know" answers were scored 0 ; thus, the score could range from 0 to 10. The questions reflected the key messages delivered in the information booklet. The questionnaire was developed by the first researcher, validated by two perioperative nurses, and pretested on eight participants during the pre- and postoperative periods. After a psychometric analysis using the Rasch scoring method, it was redesigned for application in this study population. ${ }^{[11]}$

Clinical assessment instrument was developed by the first researcher, validated by two surgeons, and pretested during the pilot study with 8 participants undergoing orthognathic surgery. ${ }^{[11]}$ Consisted of checkbox present/absent, on 7 themes. Oral hygiene (halitosis, discolored gingiva, gingivitis, coated tongue, sutures with food residues, bacterial plaque, and difficulty of performing oral hygiene). Nutrition (weight measurement, eating difficulty, and necessity for eating assistance). Mobility/sensitivity (mouth opening more limitation than expected, mandibular deviation, tactile stimulus, paresthesia, performing facial movements, use of orthodontics elastic, and difficulty of handling orthodontic elastic). Lip conditions (lip dryness, lesions, and hydration). Edema (more edema than expected, strategies for reducing edema, and facial exercises). Pain (intensity and treatment). Sleeping and breathing conditions (difficulty of sleeping, breathing, and methods to improve sleeping and breathing conditions). The score was 0 for the presence of normal (expected for the postoperative phase) and 1 for abnormal symptoms (not expected - possible complications).

\subsection{Data collection}

First contact with participants took place during the preoperative period at doctor's office. After that, enrollment and usual care were completed. During the postoperative period, collected data included knowledge, anxiety, and clinical assessments during four office visits to the surgeon on days 7 , 15,20 , and 45 after surgery. During the 45-day postoperative visit, data about facial appearance and satisfaction with outcomes (facial appearance, voice change, confidence, or others) were collected. To avoid bias, participants were assisted by a single surgical team, so that there was uniformity in the surgical technique and in the pre- and postoperative guidance provided by surgeon.

\subsection{Data analysis}

General linear modeling and linear mixed-effects model analysis were used to compare outcomes (knowledge, anxiety and clinical assessment) for intervention and control groups. Chi-square test was used to analyze the effect of demographic characteristics (age, gender, civil state, and scholarly level). F-test was applied to analyze motivation and postoperative changes. Significance was set at $p=.05$. Data analysis was conducted using SPSS 20.0 (IBM, Chicago, IL).

\section{RESULTS}

There were no differences between groups at baseline regarding gender, age, civil state, motivation for the surgery, except that control group participants were more likely to have received post high school education. The average age of the intervention group was $26.80 \pm 8.01$ years and the age of the control group was $27.40 \pm 6.79$ years $(p=.802)$. At baseline, there were no differences between groups in knowledge or anxiety (see Table 1).

Intervention provided better results for intervention group on knowledge about postoperative self-care $(p<.001)$, however there was no significant difference for state anxiety $(p=.818)$ (see Table 2).

Halitosis was higher in intervention group $(p=.003)$; this group had more difficulty with oral hygiene than control group, as $20 \%$ of participants reported fear of touching the suture and $50 \%$ could not open their mouth because of orthodontic elastics. intervention group presented higher weight loss compared with control group $(p=.002)$ (see Table 3). 
Table 1. Patients' characteristics at baseline

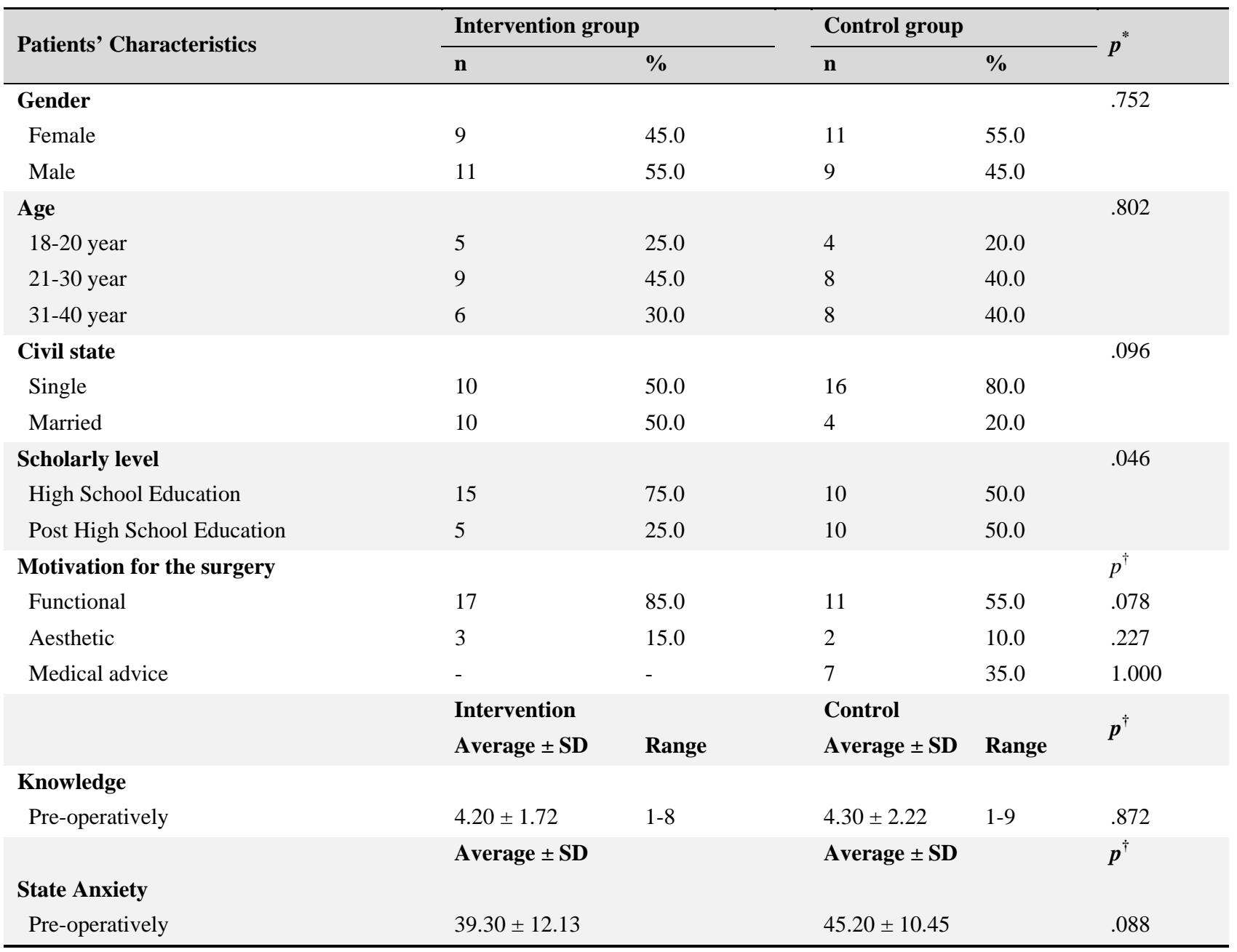

*Chi-Square test; $\uparrow F$ test; SD = standard deviation.

Table 2. Knowledge, anxiety test by groups and postoperative changes

\begin{tabular}{|c|c|c|c|c|c|c|}
\hline \multirow{3}{*}{ Assessment } & \multicolumn{4}{|l|}{ Group } & \multirow{3}{*}{$p^{*}$} & \multirow{3}{*}{$\boldsymbol{p}^{\dagger}$} \\
\hline & \multicolumn{2}{|l|}{ Intervention } & \multicolumn{2}{|l|}{ Control } & & \\
\hline & Average \pm SD & Range & Average \pm SD & Range & & \\
\hline \multicolumn{7}{|l|}{ Knowledge } \\
\hline Post-op $1^{\text {st }}$ visit & $6.50 \pm 1.53$ & $4-10$ & $4.30 \pm 1.94$ & $1-8$ & .001 & \multirow{3}{*}{$<.001$} \\
\hline Post-op $3^{\text {rd }}$ visit & $6.80 \pm 1.65$ & $4-10$ & $5.00 \pm 2.21$ & $1-9$ & .005 & \\
\hline Post-op $4^{\text {th }}$ visit & $7.00 \pm 1.55$ & $3-10$ & $5.20 \pm 2.32$ & $1-9$ & .004 & \\
\hline \multicolumn{7}{|l|}{ State Anxiety } \\
\hline Post-op $1^{\text {st }}$ visit & \multirow{3}{*}{\multicolumn{2}{|c|}{$\begin{array}{l}35.80 \pm 11.37 \\
33.90 \pm 9.41 \\
34.40 \pm 10.21\end{array}$}} & \multirow{3}{*}{\multicolumn{2}{|c|}{$\begin{array}{l}40.10 \pm 11.24 \\
36.70 \pm 10.39 \\
37.20 \pm 10.46\end{array}$}} & .216 & \multirow{3}{*}{.818} \\
\hline Post-op $3^{\text {rd }}$ visit & & & & & .422 & \\
\hline Post-op $4^{\text {th }}$ visit & & & & & .422 & \\
\hline Postoperative changes & n & $\%$ & $\mathbf{n}$ & $\%$ & $p^{*}$ & \\
\hline Face appearance & 14 & 70.0 & 13 & 65.0 & .431 & \\
\hline Voice & 1 & 5.0 & - & - & 1.000 & \\
\hline Chewing & 1 & 5.0 & 6 & 30.0 & .001 & \\
\hline Confidence & 4 & 20.0 & 1 & 5.0 & .005 & \\
\hline
\end{tabular}

*F tests; †Mixed-effect models; SD = standard deviation. 
Table 3. Clinical assessments by group

\begin{tabular}{|c|c|c|c|c|c|c|c|c|c|}
\hline \multirow{3}{*}{ Variables } & \multicolumn{8}{|c|}{ Postoperative (\%) } & \multirow{3}{*}{$p^{*}$} \\
\hline & \multicolumn{2}{|l|}{$1^{\text {st }}$ visit } & \multicolumn{2}{|c|}{$2^{\text {nd }}$ visit } & \multicolumn{2}{|l|}{$3^{\text {rd }}$ visit } & \multicolumn{2}{|l|}{$4^{\text {th }}$ visit } & \\
\hline & Int & Con & Int & Con & Int & Con & Int & Con & \\
\hline \multicolumn{10}{|l|}{ Oral hygiene } \\
\hline Halitosis & 40.0 & 25.0 & 25.0 & 5.0 & 5.0 & - & - & - & .003 \\
\hline Discolored gingiva & - & - & - & - & - & - & - & - & - \\
\hline Gingivitis & 5.0 & 10.0 & 5.0 & - & 5.0 & - & - & - & .241 \\
\hline Coated tongue & 60.0 & 60.0 & 50.0 & 55.0 & 20.0 & 15.0 & 5.0 & 15.0 & .444 \\
\hline Suture with food residues & 35.0 & 20.0 & 20.0 & 15.0 & 5.0 & 5.0 & - & - & 1.000 \\
\hline Presence of bacterial plaque & 15.0 & 15.0 & 15.0 & - & 15.0 & - & 5.0 & 10.0 & .174 \\
\hline Difficulty of performing oral hygiene & 70.0 & 60.0 & 45.0 & 30.0 & 25.0 & 5.0 & 5.0 & 5.0 & .602 \\
\hline \multicolumn{10}{|l|}{ Mobility and Sensitivity } \\
\hline Greater mouth opening limitation than expected & 5.0 & - & - & 5.0 & - & - & - & - & 1.000 \\
\hline Mandibular deviation & - & - & - & - & - & - & - & - & - \\
\hline Response to tactile stimulus & 50.0 & 50.0 & 65.0 & 65.0 & 70.0 & 75.0 & 70.0 & 80.0 & .653 \\
\hline Performing facial movements & 5.0 & 15.0 & - & - & - & - & - & - & 1.000 \\
\hline Paresthesia & 100.0 & 90.0 & 90.0 & 90.0 & 85.0 & 85.0 & 85.0 & 85.0 & .205 \\
\hline \multicolumn{10}{|l|}{ Lip Hydration } \\
\hline Lip dryness & 70.0 & 55.0 & 55.0 & 45.0 & 40.0 & 15.0 & 30.0 & 10.0 & .543 \\
\hline Lip lesion & 15.0 & 15.0 & 10.0 & 10.0 & 10.0 & 5.0 & - & 5.0 & .412 \\
\hline \multicolumn{10}{|l|}{ Evolution and edema } \\
\hline More edema than expected & - & - & - & - & - & - & - & - & - \\
\hline Facial exercise & 20.0 & 15.0 & 50.0 & 50.0 & 55.0 & 55.0 & 50.0 & 35.0 & .730 \\
\hline \multicolumn{10}{|l|}{ Sleeping and breathing conditions } \\
\hline Difficulty in sleeping & 20.0 & 20.0 & 15.0 & 10.0 & 10.0 & - & 5.0 & - & .738 \\
\hline Difficulty in breathing & 15.0 & 15.0 & 10.0 & 10.0 & - & - & - & - & 1.000 \\
\hline Used some method to sleep or breathe better & 15.0 & 10.0 & 10.0 & 10.0 & 10.0 & 5.0 & 10.0 & 5.0 & .766 \\
\hline \multicolumn{10}{|l|}{ Nutrition (eating condition) } \\
\hline Eating assistance & 95.0 & 95.0 & 85.0 & 85.0 & 85.0 & 75.0 & 80.0 & 65.0 & .520 \\
\hline Eating difficulty & 35.0 & 55.0 & 15.0 & 30.0 & 5.0 & 15.0 & 5.0 & - & .382 \\
\hline \multicolumn{10}{|l|}{ Pain (treatment) } \\
\hline \multirow[t]{2}{*}{ Did treatment } & 35.0 & 30.0 & 15.0 & 5.0 & 5.0 & 10.0 & 5.0 & - & .217 \\
\hline & \multicolumn{8}{|c|}{ Postoperative, Average \pm SD } & $p \dagger$ \\
\hline \multicolumn{10}{|l|}{ Nutrition (change in body weight-kg-from baseline) } \\
\hline Weight & $\begin{array}{l}1.95 \pm \\
2.47\end{array}$ & $\begin{array}{l}0.30 \pm \\
2.32\end{array}$ & $\begin{array}{l}1.95 \pm \\
2.56\end{array}$ & $\begin{array}{l}0.25 \pm \\
2.13\end{array}$ & $\begin{array}{l}2.20 \pm \\
2.63\end{array}$ & $\begin{array}{l}0.50 \pm \\
2.39\end{array}$ & $\begin{array}{l}2.75 \pm \\
2.73\end{array}$ & $\begin{array}{l}0.15 \pm \\
2.28\end{array}$ & .002 \\
\hline \multicolumn{10}{|l|}{ Pain (visual analog scale) } \\
\hline Intensity & $\begin{array}{l}2.10 \pm \\
2.70\end{array}$ & $\begin{array}{l}0.70 \pm \\
1.30\end{array}$ & $\begin{array}{l}0.80 \pm \\
1.60\end{array}$ & $\begin{array}{l}0.50 \pm \\
1.50\end{array}$ & $\begin{array}{l}0.60 \pm \\
1.30\end{array}$ & $\begin{array}{l}0.60 \pm \\
1.50\end{array}$ & $\begin{array}{l}0.00 \pm \\
0.20\end{array}$ & $\begin{array}{l}0.00 \pm \\
0.20\end{array}$ & .063 \\
\hline
\end{tabular}

*Generalized linear models; †Mixed-effect models; Int = Intervention, Con = Control; SD = standard deviation.

At the end of the study, participants from both groups re- baseline to last follow-up period for the whole sample. ported being satisfied with the outcomes of surgery, and mentioned significant changes in terms of physical appearance. Results demonstrated significant difference for better chewing $(p=.001)$ and higher confidence $(p=.005)$ from

\section{DiscuSSION}

Higher-level knowledge in intervention group indicated the effectiveness of the educational intervention for learning the 
postoperative care. This result was similar to other study with booklet intervention and surgeon oral guidance in orthopaedic patients. ${ }^{[12]}$

A study conducted in Jordan ${ }^{[13]}$ assessed the perception of patients undergoing orthognathic surgery. The results showed that the patients believed that preoperative information was adequate; however, they suggested the use of additional technologies, including leaflets or videos in order to process the received data and meet the perioperative nurses before undergoing surgery.

Halitosis and weight loss were significantly different between the groups. Orthodontic elastics in intervention group was higher than in control group. The use of orthodontic elastics contributes to high difficulty in opening the mouth, this resulted in difficulty for eating and performed the oral hygiene. Consequently, there were more chances of patient present halitosis and weight loss.

Halitosis is a common term to describe unpleasant or offensive odor emanating from the oral cavity. The physio pathological causes were related to poor oral hygiene during the postoperative period-are tongue coating, food impaction in interproximal spaces, orthodontic brackets, wires, springs, screws, bands, elastic, and the surgical wound increase halitosis. ${ }^{[14,15]}$

Oral hygiene advice should be given verbally, accompanied by written directions for future reference. It is advisable to demonstrate cleaning methods with visual a fixed appliance can be a useful tool. ${ }^{[15]}$ In this study the oral hygiene demonstration was not applied, only verbal and written guidance. For further research, demonstrate the methods of performing oral hygiene should be applied to evaluate the results for halitosis.

Participants of the both groups related fear of manipulating the newly operated area, difficulty with the edema, and mouth opening limitation. Study conducted in Brazil ${ }^{[16]}$ that assessed the perception/knowledge of patients after orthognathic surgery reported their difficulty in performing oral hygiene and eating properly. It is assumed that patients may be afraid of moving the lips and cheeks and see or keep contact with the suture. Consequently, precarious oral hygiene may predispose the presence of food residues in the oral cavity, halitosis, gingivitis, and infection.

Weight loss during postoperative period had also been observed in another studies. ${ }^{[17,18]}$ Patients undergoing orthognathic surgery experience some degree of weight loss, which can not to be related to age, type and duration of surgery, length of hospital stay, and use of intermaxillary fixation. ${ }^{[19]}$ In retrospective study with 4,487 patients a significant dif- ference in weight loss experience was seen between genders, reported nutritional imbalance plus weight and fat losses should be expected in female patients. ${ }^{[19]}$ This results was different this study, the intervention group had more male patients and present more weight loss.

It was recommended that participants understand the need for a light, nutritious, and high-calorie diet after the surgery to maintain their body weight, and consequently, accelerate recovery. ${ }^{[8,17]}$ In the booklet used for this intervention, it was emphasized the importance of nutritious feeding and with adequate consistency for postoperative period. To reinforce the information, a highlighted table showed the food consistency allowed in each phase of the postoperative period.

Further investigation is needed to assess the actual postoperative weight loss and changes in nutritional status in orthognathic surgery and the relationship between changes in body weight, nutritional status and postoperative complications.

Complications after orthognathic surgery involve infection, nerve injuries, temporomandibular joint disorder, and cicatrization problems. ${ }^{[20]}$ Perioperative nurse implement strategies to better assist patients with oral hygiene, weight loss, and possible complications, and they also develop research, promote evidence-based best practices for care of these patients.

There were some limitations of the study. The sample size was small; it may have been too small to measure differences between groups, especially for factors that did not occur with high frequency. To truly understand the value of an educational booklet, largest sample should be used in future research, as outcomes in this study were from a single center. Findings from this study may not be generalizable to other oral surgery centers, because this was the small sample from one outpatient clinic, the other countries or clinics maybe find different results. Further, the booklet may not have been valuable if routine oral guidance given by doctors superceded content of the booklet and guidance by nurses or if content was too similar and only reinforced content already learned. Assessments were carried out during routine postoperative visits to the surgeon; the short time frame between assessments and repeated use of the same tools could have created threats to internal validity of findings, because the short time for repeat evaluation could have not represent the time of adequate recuperation. better results could have analyzed with more space of time, six months or one year after the surgery.

\section{Conclusion}

The booklet intervention improved patient knowledge, however knowledge did not translate into differences between 
groups in anxiety and other factors. Weight loss and halitosis were more prevalent in the intervention group than in the control group patients. The participants with the orthodontic elastics had greater difficulty in terms of mouth opening and difficulty for performed oral hygiene. The perioperative nurse should reinforce the need to perform oral hygiene in the postoperative period and reassure the patient about the difficulty of opening the mouth and the handling of orthodontic elastics for better results for halitosis and weight loss.

Finally, we recommend the use of validated booklets to assist patients undergoing orthognathic surgery in order to increase their knowledge about the procedure and postoperative self-care. Further studies are necessary to assess patients' adherence to booklets for self-care.

\section{CONFlicts of InTEREST Disclosure}

The authors declare that there is no conflict of interest.

\section{REFERENCES}

[1] Robinson RC, Holm RL. Orthognathic surgery for patients with maxillofacial deformities. AORN J. 2010; 92(1): 28-49; quiz 50-52. PMid:20619771 https://doi.org/10.1016/j . aorn. 2009.12 .030

[2] Bruton J, Norton C, Smyth N, et al. Nurse handover: patient and staff experiences. Br J Nurs. 2016; 25(7): 386-903. PMid:27081733 https://doi.org/10.12968/bjon.2016.25.7.386

[3] Johansson K, Nuutila L, Virtanen H, et al. Preoperative education for orthopaedic patients: systematic review. J Adv Nurs. 2005; 50(2): 212-223. PMid:15788086 https://doi.org/10.1111/j.1365 $-2648.2005 .03381 . x$

[4] McDonald S, Page MJ, Beringer K, et al. Preoperative education for hip or knee replacement. Cochrane Database Syst Rev. 2014; 13(5): CD003526.

[5] Moulton LS, Evans PA, Starks I, et al. Pre-operative education prior to elective hip arthroplasty surgery improves postoperative outcome. Int Orthop. 2015; 39(8): 1483-1486. PMid:25862634 https://doi.org/10.1007/s00264-015-2754-2

[6] Tait MA, Dredge C, Barnes CL. Preoperative patient education for hip and knee arthroplasty: financial benefit? J Surg Orthop Adv. 2015; 24(4): 246-251. PMid:26731389

[7] Suresh K. An overview of randomization techniques: An unbiased assessment of outcome in clinical research. J Hum Reprod Sci. 2011; 4(1): 8-11. PMid:21772732 https://doi.org/10.4103/0974-1 208.82352

[8] Sousa CS, Turrini RNT. Creating and validating educational material for patients undergoing orthognathic surgery. Asian Nurs Res (Korean Soc Nurs Sci). 2012; 6(4): 166-172. PMid:25031119 https://doi.org/10.1016/j.anr.2012.10.006

[9] Sousa CS, Turrini RNT. Construct validation of educational technology for patients through the application of the Delphi technique. Acta Paul Enferm. 2012; 25(6): 990-996.

[10] Julian LJ. Measures of anxiety: state-trait anxiety inventory (STAI), beck anxiety inventory (BAI), and hospital anxiety and depression scale-anxiety (HADS-A). Arthritis Care Res (Hoboken). 2011;
63(Suppl 11): S467-S472. PMid:22588767 https ://doi .org/10 .1002 /acr. 20561

[11] Sousa CS, Turrini RNT, Poveda VB. Educational intervention in patients undergoing orthognathic surgery: pilot study. J Nurs Educ Practice. 2015; 5(5): 126-134. https://doi .org/10.5430/jnep .v5n5p126

[12] Eschalier B, Descamps S, Pereira B, et al. Randomized blinded trial of standardized written patient information before total knee arthroplasty. PLoS One. 2017; 12(7): e0178358.

[13] Ghannam SA, Malkawi ZA, Sawair FA, et al. Perception of outcome after orthognathic surgery at Jordan university hospital. Jordan Med J. 2010; 42: 1-8.

[14] Mantovani AC, Grigoleto ARL. Halitosis: a matter of public health. Braz J Health. 2012; 1(3): 186-192.

[15] Macpherson P. Oral health advice for patients with orthodontic appliances. Dental Nurs. 2015; 11(10): 600-5. https ://doi .org/10.1 2968/denn.2015.11.10.600

[16] Barros BR, Sousa CS, Turrini RNT. Knowledge of Internet-using patients about the perioperative period of orthognathic surgery. J Nurs Educ Pract. 2013; 3: 93-102.

[17] Khattak ZG, Benington PC, Khambay BS, et al. An assessment of the quality of care provided to orthognathic surgery patients through a multidisciplinary clinic. J Craniomaxillofac Surg. 2012; 40(3): 243247. PMid:21752662 https://doi.org/10.1016/j.jcms. 201 1.04 .004

[18] Hammond D, Williams RW, Juj K, et al. Weight loss in orthognathic surgery: a clinical study. J Orthod. 2015; 42(3): 220-8. PMid:25904194 https://doi.org/10.1179/1465313314Y.00 00000130

[19] Ruslin M, Dekker H, Tuinzing DB, et al. Assessing the need for a protocol in monitoring weight loss and nutritional status in orthognathic surgery based on patients experiences. J Clin Exp Dent. 2017; 9(2): e272-75.

[20] Sousa CS, Turrini RNT. Complications in orthognathic surgery; a comprehensive review. J Oral Maxillofac Surg Med Pathol. 2012; 24(2): 67-74. https://doi.org/10.1016/j.ajoms .2012.01. 014 